\title{
Modelización Econométrica de los Accidentes de Tránsito en el Ecuador
}

\author{
Castillo, Daniela $^{1}$ (D) ; Coral, Christopher ${ }^{1}$ iD ; Salazar Méndez, Yasmín 2, * iD \\ ${ }^{1}$ Escuela Politécnica Nacional, Ingeniería en Ciencias Económicas y Financieras, Quito, Ecuador. \\ ${ }^{2}$ Escuela Politécnica Nacional, Facultad de Ciencias, Departamento de Economía Cuantitativa, Quito, Ecuador.
}

\begin{abstract}
Resumen: Los accidentes de tránsito constituyen una gran problemática de salud pública en el mundo. Según estimaciones de la Organización Mundial de la Salud (OMS, 2017) anualmente mueren cerca 1.3 millones de personas por causas relacionadas con accidentes de tránsito. Adicionalmente, según el mismo organismo, anualmente, entre 20 y 50 millones de personas sufren heridas o quedan discapacitadas producto de un accidente de tránsito. En el Ecuador, los accidentes de tránsito constituyen una gran problemática económica, social y de salud pública, siendo una de las principales causas de muerte. Durante los últimos años, la tasa de fallecidos por accidentes de tránsito ha ido en aumento, convirtiendo al Ecuador en uno de los países con mayor tasa de mortalidad de América Latina. En este artículo se analizan los accidentes de tránsito desde dos enfoques: i) pronóstico de accidentes de tránsito, ii) determinantes humanos y físicos de mayor incidencia en los siniestros de tránsito considerando los cuatro tipos de vehículos más utilizados en el Ecuador: motocicletas, vehículos livianos, comerciales, de pasajeros y pesados. Para esto se estimó un modelo ARIMA siguiendo la metodología de Box-Jenkins y un modelo logit multinomial, respectivamente. Los datos fueron proporcionados por la Policía Nacional y corresponden al periodo 2015, 2016, 2017 y 2018.
\end{abstract}

Palabras clave: Accidentes de tránsito; Box-Jenkins; Modelo logit multinomial; Ecuador.

\section{Econometric Modeling of Traffic Accidents in Ecuador}

\begin{abstract}
Traffic accidents constitute a major public health problem in the world. According to estimates of the World Health Organization (WHO, 2017), nearly 1.3 million people die annually from causes related to traffic accidents. Additionally, according to the same organization, annually, between 20 and 50 million people suffer injuries or become disabled as a result of a traffic accident. In Ecuador, traffic accidents constitute a major economic, social and public health problem, being one of the main causes of death. In recent years, the death rate from traffic accidents has been increasing, making Ecuador one of the countries with the highest mortality rate in Latin America. In this article, traffic accidents are analyzed from two perspectives: i) forecast of traffic accidents, ii) human and physical determinants of greater incidence in traffic accidents considering the four types of vehicles most used in Ecuador: motorcycles, lightweight vehicles, commercial, passenger and cargo vehicles. For this, an ARIMA model was estimated following the Box-Jenkins methodology and a multinomial logit model, respectively. The data was provided by the National Police and corresponds to the period 2015, 2016, 2017 and 2018.
\end{abstract}

Keywords: Traffic accidents; Box-Jenkins; Multinomial logit model; Ecuador.

\section{INTRODUCCIÓN}

Los accidentes de tránsito constituyen una gran problemática de salud pública en el mundo. Según estimaciones de la Organización Mundial de la Salud (OMS, 2017), anualmente mueren cerca 1.3 millones de personas por causas relacionadas con accidentes de tránsito. Adicionalmente, según el mismo organismo, entre 20 y 50 millones de personas sufren heridas o quedan discapacitadas producto de un accidente de tránsito cada año.

En el Ecuador, los accidentes de tránsito son considerados la sexta causa de muerte (INEC, 2017). De acuerdo con los datos de la Dirección Nacional de Control del Tránsito y Seguridad Vial de la Policía Nacional del Ecuador, en 2018, se reportó un total de 10329 víctimas de accidentes de tránsito y, de este grupo, 1817 fallecieron. Las estadísticas presentadas por el INEC muestran que, en 2015, la tasa de fallecidos fue de $6 \%$, es decir, 1038 fallecidos de 17492 accidentes. En 2016 y 2017 , esta tasa se mantuvo entre $6 \%$ y $7 \%$. Por último, en comparación con el resto del mundo, el Ecuador tiene una tasa promedio de mortalidad de 20.1 fallecidos semanales y la media mundial es de 18 personas (INEC, 2017).

A pesar de que, en la primera mitad de la última década, la cantidad de accidentes de tránsito se redujo, -en 2010 y 2013 
se reportó un total de accidentes de 25588 y 22559 , respectivamente; en los años posteriores, esta cantidad ha ido en aumento. Así, en 2014 se suscitaron 38658 accidentes y, en 2017, esa cifra se ubicó en 26291.

En el Ecuador, la ocurrencia de accidentes distinguiendo el tipo se presenta de la siguiente manera: 1$)$ choques $(45.4 \%)$; 2) atropellos $(16.8 \%), 3)$ estrellamientos $(13.6 \%)$; 4) pérdida de pista $(10.8 \%)$ y otro tipo (13.8\%) (INEC, 2018).

En lo que respecta a las causas de los accidentes de tránsito, el $63 \%$ se debe a la falta de atención de los conductores (por imprudencia o inobservancia a las señales de tránsito) (INEC, 2018).

Un análisis realizado por el mismo organismo para determinar el tipo de vehículos más común en siniestros de tránsito muestra que el $30 \%$ pertenecen a automóviles y jeeps. La causa que justificaría este hecho es que este tipo de vehículos son los más utilizados por los ecuatorianos. En 2018, el parque automotor del Ecuador estaba constituido por 2' 403651 vehículos matriculados con la siguiente distribución: $31.2 \%$ son automóviles; $24.2 \%$ motocicletas; $18.5 \%$ camionetas; $16.5 \%$ transporte de pasajeros; y, el $9.6 \%$ son tráileres, tanqueros, volquetas, entre otros (INEC, 2018).

En este artículo se analizan los accidentes de tránsito en el Ecuador considerando dos enfoques diferentes. Primero, utilizando la metodología de Box-Jenkins se realiza un pronóstico de la cantidad de accidentes de tránsito. Adicionalmente, a través de la estimación de un modelo logit multinomial se determinan los factores humanos y físicos que inciden en la ocurrencia de siniestro de tránsito distinguiendo el tipo de vehículo. En ambos análisis se utilizaron los datos proporcionados por la Dirección Nacional de Control del Tránsito y Seguridad Vial de la Policía Nacional. Los datos tienen una periodicidad semanal y corresponden a los años 2015, 2016, 2017 y 2018.

Los hallazgos de este estudio contribuyen con información que puede auxiliar el diseño de políticas de seguridad vial. El articulo está organizado de la siguiente manera. Después de esta sección introductoria, se expone una revisión de la literatura en la sección 2. Luego, se presenta la descripción de los datos y de las metodologías usadas en el análisis. A continuación, en la sección 4 se presentan los resultados de las estimaciones de los modelos de series temporales y logit multinomial, respectivamente. Finalmente, la última sección contiene las conclusiones y recomendaciones del estudio.

\section{REVISIÓN DE LA LITERATURA}

Entre los factores que más inciden sobre la probabilidad de ocurrencia de un accidente de tránsito se mencionan a: características individuales (la edad y el sexo); ambientales y características de la vía. El tipo de vehículo también aparece entre los factores reportados por la literatura relacionada con el análisis de los accidentes de tránsito.

En lo que respecta a los factores individuales, la edad, el sexo y conductas de riesgo de los conductores serían determinantes para la ocurrencia de un accidente de tránsito. Chang y Yeh (2017), en un estudio realizado para Taiwán, sugieren que las personas más jóvenes y los hombres tienen más probabilidad de desobedecer las normas de tránsito y de subestimar los posibles riesgos de la conducción y, por tanto, individuos con estas características estarían más propensos a sufrir un accidente de tránsito.

Considerando el tipo de vehículo, las motocicletas aparecen en los primeros lugares en los accidentes de tránsito, sin embargo, este hecho estaría asociado a que corresponden a uno de los transportes más usados en algunos países. Por ejemplo, en Singapur, las motocicletas son uno de los tipos de medios de transporte más usados. Los accidentes en motocicletas estarían explicados por factores como: las características de la vía, medidas de control de tránsito y factores ambientales (como conducir en la noche o en clima lluvioso) (Hoong et al., 2012).

Los accidentes de tránsito pueden ser considerados fatales o no dependiendo de las secuelas presentes en las víctimas. Wu (2012) analiza la gravedad de las lesiones de los conductores que sufrieron un choque en las zonas urbanas y rurales de Nuevo México y examina varios factores relacionados con las características del vehículo, del entorno y comportamiento del conductor. Los resultados del autor sugieren que las malas condiciones de la vía, la presencia de múltiples curvas en una carretera y el uso de drogas por parte de los conductores aumentan la probabilidad de que un accidente de tránsito sea de tipo fatal (fallecimiento de alguna de las víctimas). Por otro lado, Yasmín (2014), en un análisis realizado para Bangladesh, determinó que la trayectoria del movimiento del vehículo, conducir excediendo los límites de velocidad, la edad del conductor y el no usar cinturones de seguridad incrementan la probabilidad de que un accidente sea de tipo fatal. Entre los factores que, según el autor, reducirían la gravedad de las lesiones de un conductor se incluyen a los controles de peatones en la vía, la presencia de redondeles y vías en buen estado.

\section{DATOS Y METODOLOGÍA}

\subsection{Datos}

La base de datos que se utilizó para esta investigación fue proporcionada por la Dirección Nacional de Control del Tránsito y Seguridad Vial de la Policía Nacional. Para realizar el pronóstico de los accidentes de tránsito se utilizó la cantidad de accidentes semanales suscitados durante los años 2015, 2016, 2017 y 2018. Para el análisis de los determinantes de la ocurrencia de un accidente de tránsito se trabajó únicamente con los datos del año 2018.

El registro de la información de los accidentes de tránsito se realiza en el Formulario de Registro de Accidentes de Tránsito (FRAT). Este formulario tiene como propósito registrar los datos relacionados con los accidentes de tránsito que ocurren en el país.

El levantamiento de la información se efectúa en el momento que ocurre el accidente; para esto, se procede a levantar un parte policial que legitima la ocurrencia del siniestro; si, en el instante del accidente, los entes de control no disponen de un sistema de almacenamiento ingresan y validan la información en el Sistema Nacional de Estadísticas de Tránsitos (SINET). 
Previo a la estimación de los modelos se efectuó la depuración de la base de datos con el fin de identificar datos perdidos. La presencia de estas observaciones con información faltante se debe a que, una vez suscitado el accidente de tránsito, para realizar el registro y levantamiento de la información por parte de los agentes de tránsito de la Dirección Nacional de Control de Tránsito y Seguridad Vial puede transcurrir el tiempo suficiente como para que los implicados en el accidente puedan darse a la fuga.

\subsection{Modelos Autorregresivos de medias móviles}

Los modelos autorregresivos de medias móviles, conocidos como la metodología de Box-Jenkins, se basan en que cada variable $y_{t}$ se explica por sus valores pasados o rezagados y por los términos de error estocástico. La metodología de BoxJenkins es una de las más populares para la modelización econométrica de series temporales (Gujarati y Porter, 2009).

Según Enders (2015) un modelo autorregresivo de media móvil (ARMA) tiene la forma:

$$
y_{t}=a_{0}+\sum_{i=1}^{p} a_{i} y_{t-i}+\sum_{i=0}^{q} \beta_{i} \varepsilon_{t-i}
$$

Donde: $\beta_{0}=1$.

La parte autorregresiva del modelo corresponde al término que contiene los rezagos de $y_{t}$, mientras que, la parte de media móvil son los rezagos del error contemporáneo. Si las partes autorregresiva y media móvil contienen $p$ y $q$ rezagos, respectivamente, el modelo se denomina $\operatorname{ARMA}(p, q)$.

Si las raíces características de la ecuación (1) están dentro del círculo unitario, se dice que $\left\{y_{t}\right\}$ es un modelo ARMA de $y_{t}$. Esto implica que la parte autorregresiva es estacionaria y la parte de media móvil invertible. Si una o más de las raíces características de la ecuación (1) están fuera del círculo unitario, se dice que $\left\{y_{t}\right\}$ es un proceso integrado de (1) y se denomina modelo autorregresivo integrado de medias móviles integrado (ARIMA). En este caso los parámetros son $(p, d, q)$, donde $d$ representa el número de veces que se debe diferenciar la serie para volverla estacionaria.

Para la modelización de (1) se utiliza la metodología de BoxJenkins, misma que considera cuatro etapas:

1. Identificación: se encuentran los valores adecuados de $p$ y $q$. Para esto se utilizan la función de autocorrelación simple (fas) y la función de autocorrelación parcial (fap) y los correlogramas, es decir, los gráficos de fas y fap. Para sintetizar el comportamiento de fas y fap que permiten identificar los valores p y q se considera lo siguiente:

$A R(p)$ : se observa una disminución exponencial en los valores de los rezagos de la fas y un corte abrupto en el rezago $j=p$ de fap.

MA(q): en fas los picos son significativos hasta $j=q$ y fap tiene una disminución exponencial.
ARMA (p, q): se observa un decrecimiento exponencial en los rezagos de fas y fap.

Este comportamiento se observará únicamente en series estacionarias. Si esta es no estacionaria, se debe convertirla en estacionaria antes de aplicar la metodología, es decir, esta debe ser diferenciada. El análisis de estacionariedad de una serie se puede realizar con los correlogramas y con las pruebas de raíz unitaria de Dickey-Fuller (DF) y Phillips Perron (PP).

2. Estimación de los parámetros del modelo elegido: una vez identificados los valores $p$ y $q$ se estima el modelo. La estimación se realiza por métodos de estimación no lineal (en parámetros).

3. Diagnóstico: para verificar si los residuos del modelo estimado son de ruido blanco; si no lo son, se debe regresar a la etapa 1 y proponer un nuevo modelo.

4. Pronóstico: consiste en realizar un pronóstico para los valores futuros de la serie $y_{t}$.

\subsection{Modelo logit multinomial}

Los modelos de regresión con respuesta cualitativa contienen variables dependientes que no son cuantitativas. Cuando las variables de respuesta son de tipo categórico, pero sin considerar ninguna jerarquía, es decir son de tipo nominal, se usan los modelos logit multinomial (o probit).

La metodología que se explica a continuación se basa en Greene (2002). Suponga que un individuo puede elegir entre $j$ opciones: $a, b$ y $c$, no ordenadas. Las diferentes elecciones se representan por:

$$
\begin{aligned}
& y_{a}=x^{\prime}{ }_{a} \beta_{a}+\varepsilon_{a} \\
& y_{b}=x^{\prime}{ }_{b} \beta_{b}+\varepsilon_{b} \\
& y_{c}=x^{\prime}{ }_{c} \beta_{c}+\varepsilon_{c}
\end{aligned}
$$

Donde: $\left\{\varepsilon_{a}, \varepsilon_{b}, \varepsilon_{c}\right\}$ son los efectos asociados a la decisión $y$, no observados.

$\mathrm{Si} x^{\prime}{ }_{a} \beta_{a}+\varepsilon_{a} \geq \max \left\{x^{\prime}{ }_{b} \beta_{b}+\varepsilon_{b}, x^{\prime}{ }_{c} \beta_{c}+\varepsilon_{c}\right\}$, entonces, $y=0$.

Si $x_{b}^{\prime} \beta_{b}+\varepsilon_{b} \geq \max \left\{x^{\prime}{ }_{a} \beta_{a}+\varepsilon_{a}, x^{\prime}{ }_{c} \beta_{c}+\varepsilon_{c}\right\}$, entonces, $y=1$.

Si $x_{c}^{\prime} \beta_{c}+\varepsilon_{c} \geq \max \left\{x^{\prime}{ }_{a} \beta_{a}+\varepsilon_{a}, x^{\prime}{ }_{b} \beta_{b}+\varepsilon_{b}\right\}$, entonces, $y=2$.

Existen dos efectos no observados independientes:

$$
\begin{aligned}
& \varepsilon_{01}=\varepsilon_{a}-\varepsilon_{b} \\
& \varepsilon_{02}=\varepsilon_{a}-\varepsilon_{c}
\end{aligned}
$$


Definiendo:

$$
\begin{aligned}
& x^{\prime}{ }_{a} \beta_{a}-x^{\prime}{ }_{b} \beta_{b}=x^{\prime} \beta_{01} \\
& x^{\prime} \beta_{a}-x^{\prime}{ }_{c} \beta_{c}=x^{\prime} \beta_{02}
\end{aligned}
$$

Entonces:

$$
\begin{aligned}
& \operatorname{Pr}(y=0 \mid x)=\operatorname{Pr}\left(x^{\prime} \beta_{01}+\varepsilon_{01} \geq 0, x^{\prime} \beta_{02}+\varepsilon_{02} \geq 0 \mid x\right) \\
& \quad=\operatorname{Pr}\left(\varepsilon_{01} \geq-\left(x^{\prime} \beta_{01}\right), \varepsilon_{02} \geq-\left(x^{\prime} \beta_{02}\right) \mid x\right)
\end{aligned}
$$

Se debe modelar la probabilidad de que un individuo de características $x$ pertenezca a la categoría $j: \operatorname{Pr}(\mathrm{y}=\mathrm{j} \mid \mathrm{x})$. el modelo logit multinomial se expresa como:

$$
\begin{gathered}
\operatorname{Pr}(\mathrm{y}=0 \mid \mathrm{x})=1-\operatorname{Pr}(\mathrm{y}=1 \mid \mathrm{x})-\operatorname{Pr}(\mathrm{y}=2 \mid \mathrm{x}) \\
\operatorname{Pr}(y=1 \mid x)=\frac{\exp \left(x^{\prime} \beta_{1}\right)}{1+\exp \left(x^{\prime} \beta_{1}\right)+\exp \left(x^{\prime} \beta_{2}\right)} \\
\operatorname{Pr}(y=2 \mid x)=\frac{\exp \left(x^{\prime} \beta_{2}\right)}{1+\exp \left(x^{\prime} \beta_{1}\right)+\exp \left(x^{\prime} \beta_{2}\right)}
\end{gathered}
$$

Después de la estimación se deben realizar pruebas para verificar su correcta especificación. Los análisis realizados en este trabajo son: variable omitida (RESET) y heteroscedasticidad (Breush-Pagan).

\section{RESULTADOS}

\subsection{Pronóstico del número de accidentes de tránsito}

Para pronosticar el número de accidentes de tránsito se estima un modelo usando la metodología de Box- Jenkins.

\subsubsection{Identificación}

La Figura 1 muestra la serie de accidentes de tránsito semanales desde, periodo 2015 - 2018. En la Figura 1 se puede observar una tendencia decreciente a lo largo del periodo observado. Con respecto al análisis de estacionariedad, en la Tabla 1 se presentan los resultados de las pruebas de raíz unitaria de Dickey-Fuller y Phillips-Perron. Aunque el p-valor de la prueba DF permite rechazar al $1 \%$ la hipótesis nula (la serie es no estacionaria), el p-valor de la prueba PP lo hace al $5 \%$, por tanto, se procedió a diferenciar la serie. Posteriormente, el análisis realizado a la serie diferenciada sugiere que esta ya es estacionaria y este resultado se corrobora por las dos pruebas (DF y PP).

Tabla 1. Pruebas de Raíz Unitaria

\begin{tabular}{crrrr}
\hline \multicolumn{5}{c}{ Variable Número de Accidentes Semanales } \\
\hline \hline \multirow{2}{*}{ Prueba } & \multicolumn{2}{c}{ Variable sin Diferenciar } & \multicolumn{2}{c}{ Variable Diferenciada } \\
& Coeficiente Probabilidad & Coeficiente & Probabilidad \\
\hline Dickey-Fuller & -11.87 & 0.0000 & -4.249 & 0.0005 \\
Phillips-Perron & -24.78 & 0.0560 & -245.616 & 0.0000 \\
\hline
\end{tabular}

Elaborado por: Los Autores

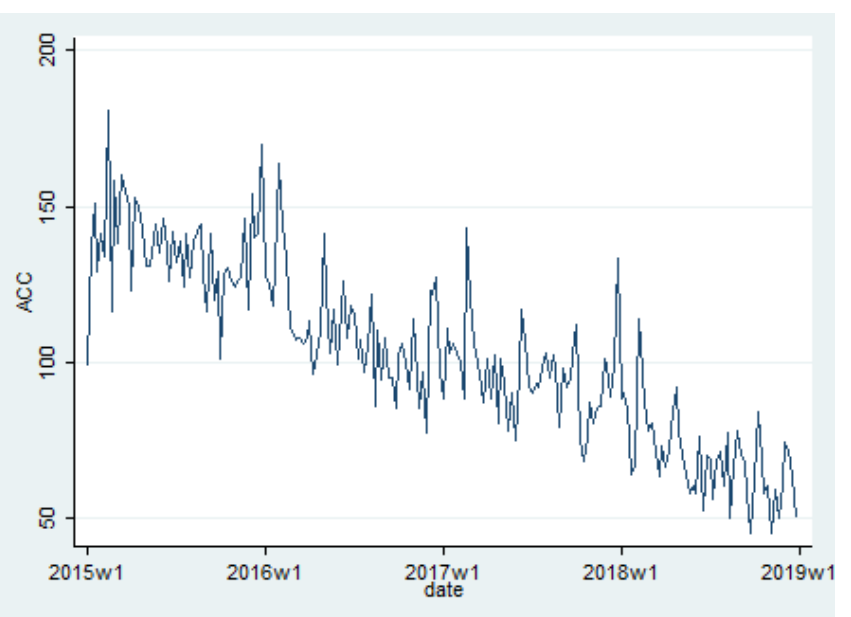

Figura 1. Número de Accidentes de Tránsito por semana 2015-2018 Fuente: Policía Nacional

En el correlograma de la serie diferenciada, ver la Figura 2, se observa que las funciones de autocorrelación simple y parcial tienen un decrecimiento exponencial hacia cero y, en el caso de fas también se puede evidenciar un corte abrupto. Por lo expuesto, se especifican los siguientes modelos ARIMA: ARIMA $(1,1,0), \operatorname{ARIMA}(1,1,1)$ y ARIMA $(0,1,1)$.
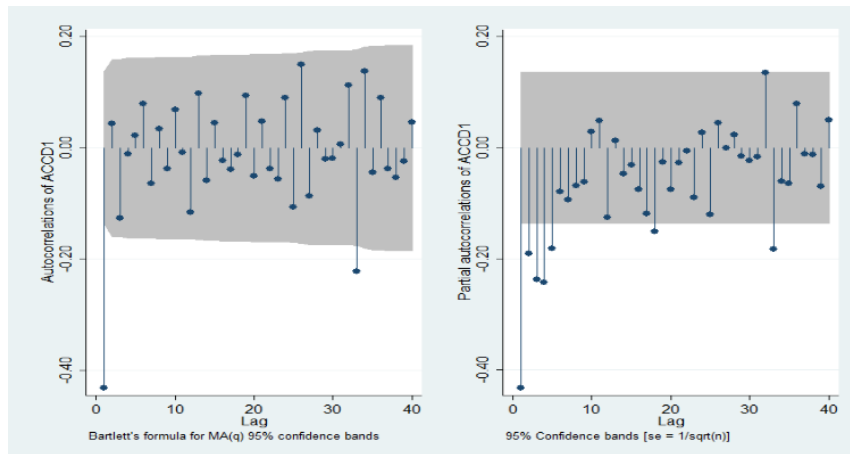

Figura 2. Funciones de autocorrelación simple y parcial de la serie diferenciada

Elaborado por: los Autores

\subsubsection{Estimación}

\begin{tabular}{|c|c|c|c|c|}
\hline \multicolumn{5}{|c|}{ Varible Dependiente Diferenciada: Número de Accidentes } \\
\hline Modelo & $\mathbf{A R}(\mathbf{1})$ & $\mathbf{M A}(\mathbf{1}$ & & ARMA(1,1) \\
\hline Variable & Coeficiente & Coeficiente & & Coeficiente \\
\hline L1.ar & $-0.4414 * * *$ & & & $0.2267 * * *$ \\
\hline L1.ma & & -0.8844 & $* * *$ & -1.0000 \\
\hline Muestra & 207 & 207 & & 207 \\
\hline BIC & 1739.51 & 1698.662 & & 1696.286 \\
\hline AIC & 1729.51 & 1688.664 & & 1682.956 \\
\hline
\end{tabular}

Los resultados de los modelos estimados se muestran en la Tabla 2. La selección del mejor modelo se hizo considerando el criterio de información de Akaike (AIC), dando como resultado que la mejor especificación del modelo corresponde al modelo ARIMA $(1,1,1)$.

Tabla 2. Resultados de las estimaciones

Nota: * $\mathrm{p}<0.10, * * \mathrm{p}<0.05, * * * \mathrm{p}<0.01$

Elaborado por: Los Autores 


\subsubsection{Diagnóstico}

En la Figura 3 se muestra el correlograma de los residuos del modelo propuesto y se puede concluir que los residuos son ruido blanco. Las barras de las funciones de autocorrelación simple y parcial son estadísticamente nulas. Adicionalmente, esto se confirma a través de la prueba de Portmanteau, obteniendo un p-valor de 0.3921 que, no permite rechazar la hipótesis nula de que los residuos siguen un proceso de ruido blanco.

En cuanto a la normalidad de los residuos, las pruebas de Jarque Bera y Shapiro Wilk evidencian normalidad en los residuos. Para verificar la autocorrelación se aplicó la prueba de Breusch Godfrey, el p-valor obtenido es de 0.4249 que no permite rechazar la hipótesis nula, de este modo se concluye que no existe autocorrelación en los residuos.
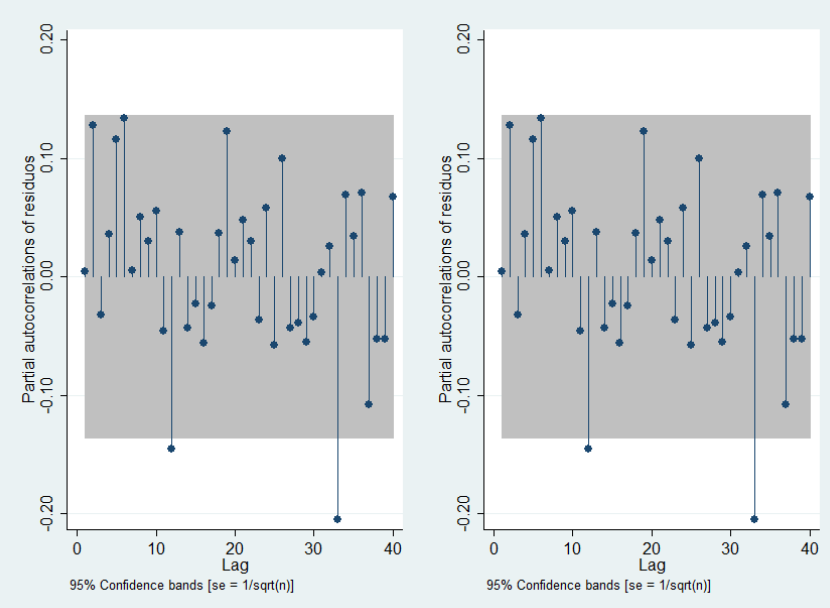

Figura 3. Correlograma de los residuos del Modelo ARIMA $(1,1,1)$ Elaborado por: los Autores

Finalmente, en la Figura 4, se muestra el ajuste del modelo estimado considerando los valores observados y los valores pronosticados. Se puede observar que, en general, el modelo presenta un buen ajuste.

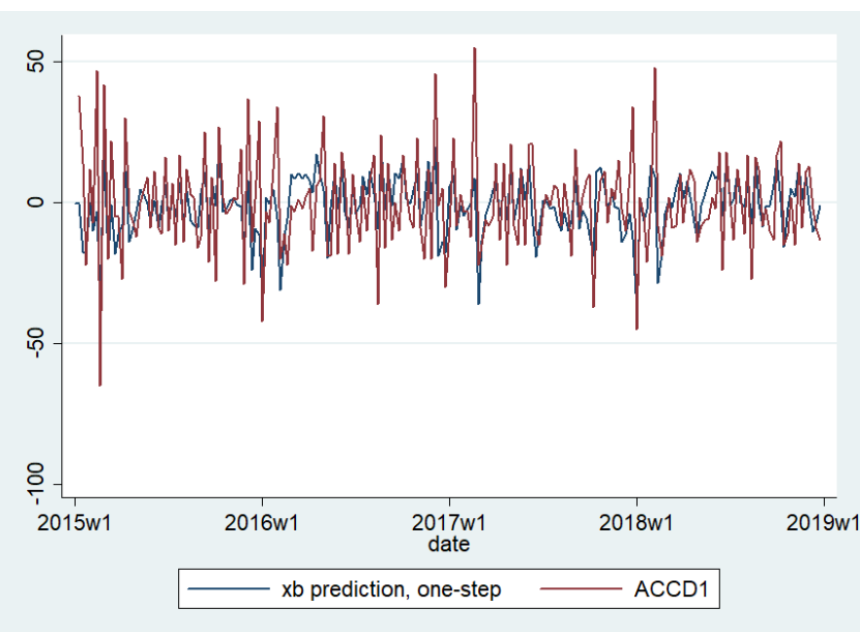

Figura 4. Ajuste del modelo $\operatorname{ARIMA}(1,1,1)$ Elaborado por: los Autores

\subsubsection{Pronóstico}

Para pronosticar el número de accidentes de tránsito se utilizó el modelo ARIMA $(1,1,1)$. En el pronóstico de los accidentes de tránsito de las semanas: 1, 2, 3 y 4 para el año 2019; se observa un leve crecimiento. Los valores pronosticados de accidentes de tránsito para dichas semanas son:

- Primera Semana: 57 accidentes

- Segunda Semana: 59 accidentes

- Tercera Semana: 59 accidentes

- Cuarta Semana: 58 accidentes

Según los datos registrados por la Dirección Nacional de Control del Tránsito y Seguridad Vial de la Policía Nacional, los siniestros de tránsito para las cuatro primeras semanas del 2019 fueron:

- Primera Semana: 60 accidentes

- Segunda Semana: 63 accidentes

- Tercera Semana: 67 accidentes

- Cuarta Semana: 55 accidentes

\subsection{Modelo logit multinomial}

Para determinar los factores que inciden en la probabilidad de ocurrencia de un accidente de tránsito, considerando los distintos tipos de vehículos, se estimó un modelo logit multinomial. La selección de la metodología se justifica por la naturaleza de la variable dependiente, misma que contiene cinco categorías: motocicletas, vehículos livianos, vehículos comerciales, vehículos de pasajeros y vehículos de transporte de carga pesada. La categoría "livianos" está conformada por automóviles y jeeps, la categoría "comercial" abarca camionetas y vehículos que son de uso comercial, la categoría "pasajeros" incluye autobuses; y, por último, la categoría "pesado" engloba tráileres, volquetas, unidades de carga, remolques y camiones. Para el análisis se toma como categoría base a los vehículos livianos.

De acuerdo con la estadística descriptiva de la variable, en 2018, 7438 vehículos se vieron involucrados en un accidente de tránsito. De esta cantidad, $34 \%$ son automóviles o "vehículos livianos"; seguido por las camionetas y vehículos de uso comercial (31\%). Los vehículos de transporte de pesado representan el $15 \%$, mientras que, las motocicletas tienen una participación del $12 \%$. Finalmente, los accidentes con vehículos de pasajeros representan el $8 \%$.

Las variables independientes contienen características físicas y humanas: edad, sexo, estado de la persona, condición de la persona, clima, zona, accidente, causa del accidente.

Conforme con los datos proporcionados por la Dirección Nacional de Control del Tránsito y Seguridad Vial para el 2018, las personas registradas fueron 8365 , donde la edad de las personas involucradas en un siniestro de tránsito comprende desde los cero años, hasta los 99 años. En promedio, la edad de las personas involucradas en un accidente de tránsito es de 35 años. El porcentaje de mujeres que sufrieron un accidente de tránsito es de $13 \%$ y el $87 \%$ son hombres. Ver la Figura 5. Por otro lado, se observa que la mayor parte de los individuos están en los siguientes rangos de edad: 20 - 30 años, 30 - 40 años; y, 40 - 50 años. 
El estado de las personas luego del accidente de tránsito muestra que el $34 \%$ de los involucrados, salieron ilesos; el 44 $\%$ presentó alguna herida; mientras que el $22 \%$ fallecieron.

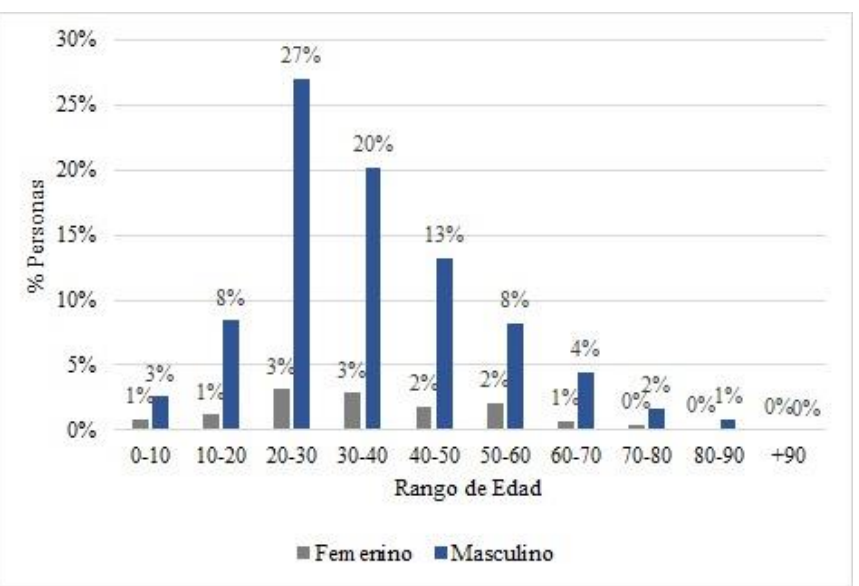

Figura 5. Personas involucradas en un siniestro por sexo y edad Elaborado por: los Autores

De acuerdo con la condición de la persona se observa que el $65 \%$ eran conductores; el $23 \%$ pasajeros; y, $12 \%$ peatones y/o testigos.

En los 4208 accidentes registrados por la Dirección Nacional de Control del Tránsito y Seguridad Vial de la Policía Nacional en 2008, se registra que el $88 \%$ acontecieron en un clima despejado; el $10 \%$ en un clima lluvioso; y, el $2 \%$ en un clima nublado. El porcentaje de accidentes de acuerdo con el estado de la vía es: $5 \%$ en adoquinado; $80 \%$ en asfalto; y, $15 \%$ en vías empedradas, lastradas y/o tierra. El porcentaje de accidentes que sucedieron en las áreas urbana y rural es similar, $50 \%$ en cada zona.

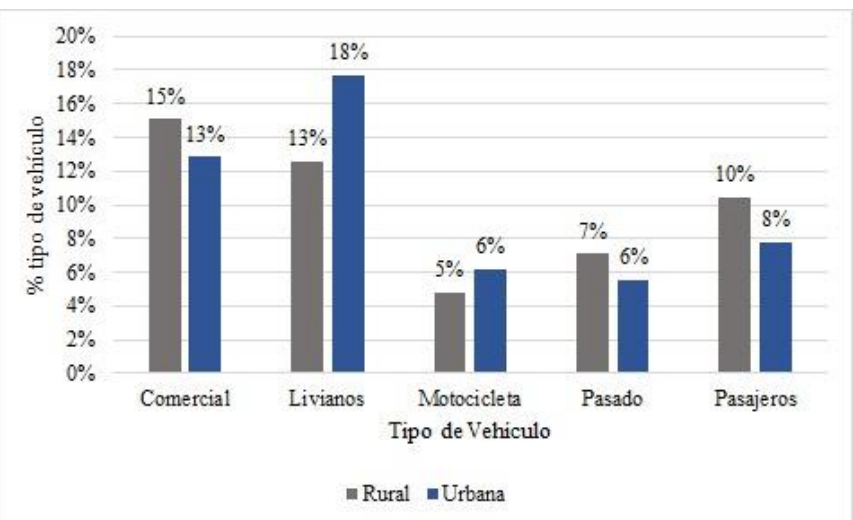

Figura 6. Tipos de vehículos involucradas en un siniestro por zona Elaborado por: los Autores

La Figura 6 indica que los vehículos livianos son los que abarcan el mayor porcentaje con un $18 \%$ de siniestros en zonas urbanas, mientras que en la zona rural esta cifra corresponde al $13 \%$. Por otro lado, se puede observar que el $15 \%$ de los vehículos comerciales estuvieron involucrados en un accidente dentro de una zona rural, mientras que, el $13 \%$ de estos siniestros se da en una zona urbana.

En cuanto a los vehículos pesados, el $6 \%$ sufrió un accidente en una zona urbana y el $7 \%$ en una zona rural. Con respecto a los vehículos de pasajeros, el $11 \%$ sufrieron accidentes en zonas rurales y el $8 \%$ en zonas urbanas. Finalmente, las motocicletas tienen un menor porcentaje, el $5 \%$ de este tipo de vehículos tuvieron un accidente de tránsito en zonas rurales en comparación al $6 \%$, que sufrieron algún tipo de accidente en zonas urbanas.

En cuanto a los tipos de accidentes, para el 2018, los atropellamientos fueron del $18 \%$; choques y colisiones fueron del $57 \%$; estrellamientos del $7 \%$; pérdidas de pista (12\%) y otros tipos del $6 \%$. La principal causa de los accidentes fue la falta de atención al conducir con $69 \%$. El resto de los accidentes corresponden a causas relacionadas con factores humanos viales y mecánicos.

Posterior a la estimación de los parámetros se realizó una serie de pruebas para garantizar una correcta especificación del modelo. Aplicando la prueba de RESET se encontró un problema de variable omitida, sin embargo, después de analizar los signos de los parámetros obtenidos se puede pensar que no hay un problema de sesgo debido a que el efecto obtenido en las estimaciones está acorde con la evidencia empírica. La prueba de Breush-Pagan arrojó la presencia de heteroscedasticidad, se corrigió este efecto estimando con errores robustos obteniendo estimadores insesgados y consistentes. Para verificar la significancia estadística de las variables se realizó la prueba de Walt, la cual muestra que todas las variables en conjunto son significativas; las variables explicativas tienen un efecto significativo en la probabilidad de ocurrencia de un accidente.

Con respecto al análisis de los resultados, que muestra la Tabla 3 , se puede observar para el caso de los hombres es más probable que sufran un accidente de tránsito en motocicletas y vehículos de carga pesada en comparación a un automóvil.

Una vez suscitado el accidente de tránsito existe una mayor probabilidad de que la persona involucrada presente una herida cuando se moviliza en una motocicleta que cuando va en un automóvil. Además, existe una menor probabilidad de fallecer en un accidente cuando el accidente sucede en un automóvil en comparación con los demás vehículos.

En cuanto a la condición de la persona, los pasajeros que se movilizan en motocicleta tienen menor probabilidad sufrir un siniestro de tránsito que en un automóvil, sin embargo, existe una mayor probabilidad de sufrir un siniestro movilizándose en vehículos comerciales, en vehículos de pasajeros y en vehículos de transporte pesado.

En relación con las causas, los factores humanos como estado de embriaguez, exceso de velocidad o imprudencia del peatón, tiene una mayor probabilidad de causar un accidente de tránsito en los vehículos livianos que en el resto de las categorías, no obstante, los accidentes en motocicleta tienen una menor probabilidad de ser producto de daños mecánicos o daños en la vía en comparación a los demás vehículos.

Según los tipos de accidentes de tránsito, un atropello tiene menor probabilidad de ser provocado por los vehículos de pasajero, comerciales o vehículos pesados en contraste con los automóviles, no obstante, los automóviles tienen mayor probabilidad de sufrir un estrellamiento que los demás vehículos. Por otro lado, los vehículos de carga pesada tienen 
una mayor probabilidad de sufrir un siniestro por pérdida de pista que los automóviles.

Considerando el clima, las motocicletas tienen menor probabilidad de ser partícipes de un accidente de tránsito que los automóviles, sin embargo, si el clima se encuentra nublado o con neblina, los vehículos de carga pesada, transporte de pasajeros y de comercio, tienen mayor probabilidad de sufrir un accidente.

En cuanto al tipo de vía, si esta es de adoquín u hormigón, las motocicletas son menos propensas a sufrir un accidente de tránsito que los automóviles, no obstante, los vehículos de transporte pesado son más propensos y esto se debe a que este tipo de vías no son adecuadas para estos vehículos. En las vías asfaltadas es más probable que los vehículos de transporte de carga y de pasajeros sufran un siniestro de tránsito en comparación con los vehículos livianos.

Distinguiendo las áreas, entre urbana y rural, los vehículos pesados y de pasajeros tienen una mayor probabilidad de sufrir un siniestro de tránsito en zonas rurales; esto podría explicarse por el hecho de que las condiciones del tránsito en estas zonas no son las mejores para este tipo de vehículos.

Tabla 3. Estimación del Modelo Logístico Multinomial

\begin{tabular}{|c|c|c|c|c|}
\hline Clase de Vehículo & Motocicleta & $\begin{array}{c}\text { Vehículo } \\
\text { Comercial }\end{array}$ & $\begin{array}{c}\text { Vehículo } \\
\text { Pasajeros }\end{array}$ & Pesados \\
\hline \multicolumn{5}{|c|}{ Variables de Factores Humanos } \\
\hline \multicolumn{5}{|c|}{ Sexo } \\
\hline Hombres & $0.476 * * *$ & 0.004 & 0.549 & $0.575 * * *$ \\
\hline Edad & $-0.015 * * *$ & $0.010 * * *$ & $-0.001 * * *$ & $0.011 * * *$ \\
\hline \multicolumn{5}{|l|}{ Estado de la Persona } \\
\hline Herido & $1.885 * * *$ & -0.048 & 0.827 & 0.139 \\
\hline Fallecido & $2.319 * * *$ & $0.234 * *$ & $1.605 * *$ & $0.702 * * *$ \\
\hline \multicolumn{5}{|l|}{ Condición de la Persona } \\
\hline Pasajero & $-0.669 * * *$ & $0.355 * * *$ & $0.536 * * *$ & $0.604 * * *$ \\
\hline Peatón o Testigo & $-0.944 * * *$ & 0.019 & 0.940 & $-0.352 *$ \\
\hline \multicolumn{5}{|l|}{ Causa de Accidente } \\
\hline Factores Humanos & $-0.347 * * *$ & $-0.129 *$ & $-0.280 *$ & $-0.520 * * *$ \\
\hline Vía y Daños Mecánicos & $-0.556 * *$ & -0.075 & -0.501 & -0.230 \\
\hline \multicolumn{5}{|l|}{ Tipo Accidente } \\
\hline Atropello & 0.295 & $-0.590 * * *$ & $-0.471 * * *$ & $-1.037 * * *$ \\
\hline Estrellamiento & $-0.447 * *$ & $-0.638 * * *$ & $-1.141 * * *$ & $-1.165 * * *$ \\
\hline Choque o Colisión & -0.121 & $-0.508 * * *$ & $-1.138 * * *$ & $-0.988 * * *$ \\
\hline Perdida de Pista & 0.339 & 0.017 & $-0.423 * *$ & $1.165 * * *$ \\
\hline \multicolumn{5}{|c|}{ Variables de Factores Físicos } \\
\hline \multicolumn{5}{|c|}{ Clima } \\
\hline Lluvia & $-0.320 * *$ & 0.041 & 0.090 & -0.125 \\
\hline Neblina y Nublado & -0.470 & $0.430 * *$ & $0.640 * *$ & $0.416 *$ \\
\hline \multicolumn{5}{|l|}{ Tipo de Vía } \\
\hline Adoquinado y Hormigón & $-0.320 * *$ & 0.198 & -0.091 & $0.684 * * *$ \\
\hline Asfalto & -0.470 & -0.066 & $0.262 * *$ & $0.227 *$ \\
\hline \multicolumn{5}{|l|}{ Área } \\
\hline Rural & -0.035 & $0.376 * * *$ & $0.565 * * *$ & $0.502 * * *$ \\
\hline Constante & $-1.985 * * *$ & $-0.806 * * *$ & $-2.281 * * *$ & $-2.247 * * *$ \\
\hline Número de Observaciones: & 8365 & & & \\
\hline Correctamente Clasificado: & $31.90 \%$ & & & \\
\hline Pseudo R2: & 0.075 & & & \\
\hline BIC: & -50666.489 & & & \\
\hline AIC: & 2.866 & & & \\
\hline
\end{tabular}

Tabla 4. Comparación de Modelos por Factores

\begin{tabular}{lrrr}
\multicolumn{4}{c}{ Tabla 4. Comparación de Modelos por Factores } \\
\hline & $\begin{array}{c}\text { Modelo de } \\
\text { Factores Humanos }\end{array}$ & $\begin{array}{c}\text { Modelo de } \\
\text { Factores Físicos }\end{array}$ & $\begin{array}{c}\text { Modelo de } \\
\text { ambos factores }\end{array}$ \\
\hline \hline Número de Observaciones & 8365.000 & 8365.000 & 8365.000 \\
\% de clasificación & $30.20 \%$ & $29.80 \%$ & $31.90 \%$ \\
BIC & -50864.204 & -49921.231 & -50666.489 \\
AIC & 2.876 & 2.992 & 2.866 \\
\hline
\end{tabular}

Elaborado por: Los Autores
Finalmente, se analiza el efecto por factores físico y humano. En la Tabla 4 se muestra que tanto factores humanos y físicos son significativos.

\section{CONCLUSIONES Y RECOMENDACIONES}

En este artículo se estimaron dos modelos; el primero para pronosticar el número de accidentes de tránsito y el segundo para determinar los factores que inciden en la ocurrencia de un accidente de tránsito. A partir de los hallazgos se concluye que, en general, los factores humanos y físicos tienen efecto sobre los siniestros.

Los accidentes de motocicletas tienen más probabilidad de causar heridas o la muerte a sus víctimas al compararlos con los accidentes de vehículos.

En cuanto a los factores humanos, tales como: estado de embriaguez, exceso de velocidad o imprudencia del peatón, estos aumentan la probabilidad de ocurrencia de siniestros en vehículos livianos.

Por último, los factores climáticos. La presencia de neblina aumenta la probabilidad de accidentes en vehículos de carga pesada, de comercio y transporte de pasajeros. Considerando el tipo de vía, sí esta es de hormigón, aumenta la probabilidad de accidentes para este tipo de vehículos, debido a que las llantas de estos vehículos pierden adherencia al piso. Adicionalmente, los resultados de la presente investigación reflejan que el estado de las vías es un factor clave para evitar accidentes, por tanto, los organismos responsables por el mantenimiento de las vías deben tener planes periódicos de mantenimiento de las vías

García et al. (2017), en un análisis sobre la percepción general de la seguridad vial en una ciudad ecuatoriana (Loja) encontraron que la mayoría de los encuestados discordaba de las leyes de tránsito, especialmente con las relacionadas con límites de velocidad y consumo de alcohol. Esto lleva a concluir a los autores la necesidad de implementar campañas de seguridad vial. En esta línea, las recomendaciones de este estudio también se enfocan en la importancia de la educación vial y el control en las vías orientado a revisar estos dos aspectos particulares.

Una de las limitaciones de este estudio es que la base proporcionada por la Dirección Nacional de Control de Tránsito y Seguridad Vial de la Policía Nacional del Ecuador no cuenta con información más completa de las personas accidentadas y las condiciones del accidente, así como del tipo de lesiones que tuvieron los involucrados, el nivel de educación de los accidentados, la calidad de la vía, el tipo de unión de la vía, entre otros. El contar con esta información permitiría realizar análisis más completos de los factores que intervienen en un siniestro. De esta forma, se recomienda que estos datos sean recolectados con el fin de realizar análisis más completos.

Para posteriores trabajos, se puede considerar posibles variables como: el nivel de las lesiones, historial de multas o infracciones, puntos en la licencia. 


\section{REFERENCIAS}

Chang, H.-L. (2006). Participación de accidentes de motociclistas por edad, género y conductas de riesgo en Taipei, Taiwán. Science Direct. 14,109-122

Dirección Nacional de Control del Tránsito y Seguridad Vial de la Policía Nacional del Ecuador. Base de Datos de Personas, Vía y Vehículos 2015-2019. (Julio, 2019)

Enders, W. (2015). Applied Econometric Time Series. University of Alabama. Cuarta edición. ISBN 978-1118-80856-6.

García, Y., Zárate, B., Segarra, S., González, J. (2017). Percepción general de la seguridad vial en la ciudad de Loja (Ecuador). Revista Cumbres 4 (1), 9 - 16.

Greene, W. (2002). Econometric Analysis, Cuarta Edición. Pearson Edit. ISBN 0-13-066189-9

Gujarati, D., Porter, D. (2009). Econometría, Quinta Edición. McGraw-Hill/Irwin, Inc. ISBN: 978-607-15-0294-0

Haque, M., Chor, H. y Kumar, A. (2012). Una investigación sobre choques de motocicletas de varios vehículos utilizando modelos log-lineales. Safety Science. 50, 352-362

Hoong, C., Quddus. M., \& Noland, R. (2002). Un análisis de lesiones en motocicleta y severidad de daños en vehículos usando modelos Probit ordenados. Journal of Safety Research. 33, 445-462.

INEC. (2017). Anuario de Estadísticas de Transporte ANET2017. Instituto Nacional de Estadísticas y Censos del Ecuador. Obtenido de: https://www.ecuadorencifras.gob.ec/documentos/we b-

inec/Estadisticas_Economicas/Estadistica\%20de\%2 0Transporte/2017/2017_TRANSPORTE_PRESENT ACION.pdf. (Julio, 2018).

INEC. (2018). Anuario de Estadísticas de Transporte 2018. Instituto Nacional de Estadísticas y Censos del Ecuador INEC. Obtenido de: https://www.ecuadorencifras.gob.ec/documentos/we binec/Estadisticas_Economicas/Estadistica\%20de\%2 0Transporte/2018/2018_ANET_BOLETIN.pdf (Octubre, 2019).

Montt, C., Rodríguez, N., Valencia, A., Barba, L. \& Rubio, M. (2014). Regresión funcional para predecir lesionados en accidentes del tránsito de la región de Valparaíso de Chile. Actas del Congreso Chileno de Ingeniería de Transporte. Santiago, Chile, Paper 54.

OMS. (2017). Informe sobre la situación mundial de la seguridad vial es hora de pasar a la acción. Organización Mundial de la Salud. Obtenido de: https://www.who.int/violence_injury_prevention/roa d_safety_status/report/web_version_es.pdf?ua=1

Yasmín, S. (2014). Factores que influyen en la gravedad de las lesiones de los conductores en Bangladesh. Asian Transport Studies. 3, 143-154.

Wu, Q. (2016). Análisis de la gravedad de las lesiones al conductor en accidentes de un solo vehículo en zonas rurales. Science Direct, 94, 35-45.

\section{BIOGRAFÍAS}

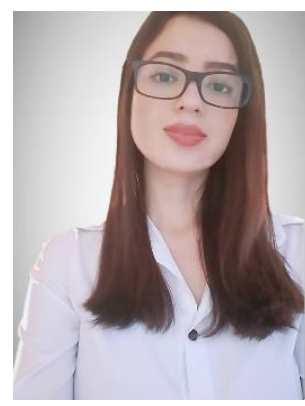

Daniela Castillo Espinoza. Estudiante de último año de la carrera de Ingeniería en Ciencias Económicas y Financieras de la Facultad de Ciencias de la Escuela Politécnica Nacional. ORCID: 9865 https://orcid.org/0000-0003-0777-

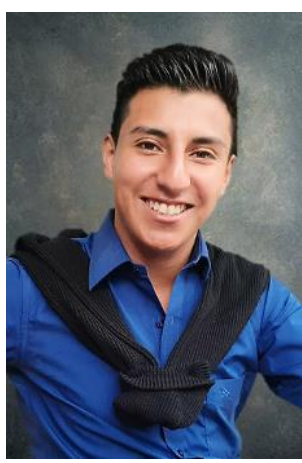

Christopher Coral Barahona. Estudiante de último año de la carrera de Ingeniería en Ciencias Económicas y Financieras de la Facultad de Ciencias de la Escuela Politécnica Nacional. Identificador ORCID: https://orcid.org/0000-0001-60812754

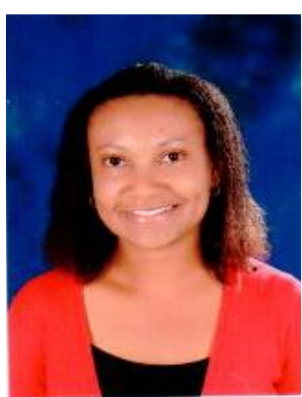

Yasmín Salazar Méndez. Desde 2015, es profesora titular de Econometría del Departamento de Economía Cuantitativa de la Facultad de Ciencias de la Escuela Politécnica Nacional. Doctora en Economía por la Universidad Federal Fluminense Brasil (2015). Obtuvo una maestría en Ingeniería de Producción en la misma universidad (2008). Ingeniera Matemática graduada en la Escuela Politécnica Nacional (2003). Su investigación se centra en métodos cuantitativos y en la economía del bienestar, con énfasis en la desigualdad económica en América Latina. Identificador digital ORCID: https://orcid.org/0000-0001-5909-9234 